\title{
A Novel Sierpinski Carpet Fractal Antenna with Improved Performances
}

\author{
Manas Ranjan Jena ${ }^{1, *}$, B.B.Mangaraj ${ }^{1}$, Rajiv Pathak $^{2}$ \\ ${ }^{1}$ Veer Surendra Sai University of Technology, Burla, Odisha \\ ${ }^{2}$ Deparment of Eltce, Bit, Chhatisgadh \\ *Corresponding author: manas.synergy@gmail.com
}

Received February 08, 2014; Revised March 12, 2014; Accepted March 16, 2014

\begin{abstract}
In modern wireless communication systems and increasing of other wireless applications, wider bandwidth, multiband and low profile antennas are in great demand for both commercial and military applications. This has initiated antenna research in various directions; one of them is using fractal shaped antenna elements. Traditionally, each antenna operates at a single or dual frequency bands, where different antenna is needed for different applications. This will cause a limited space and place problem. In order to overcome this problem, multiband antenna can be used where a single antenna can operate at many frequency bands. One technique to construct a multiband antenna is by applying fractal shape into antenna geometry. This project presents the Sierpinski carpet patch antenna where this famous shape, the antenna behaviors are investigated. In addition to the theoretical design procedure, numerical simulation was performed using software (CST) to obtain design parameters such as size of patch and feeding location. The antennas have been analyzed and designed by using the software CST Microwave Studio Suite.
\end{abstract}

Keywords: fractal, CST, IFS, multiband, VSWR, Bandwidth

Cite This Article: Manas Ranjan Jena, B.B.Mangaraj, and Rajiv Pathak, "A Novel Sierpinski Carpet Fractal Antenna with Improved Performances." American Journal of Electrical and Electronic Engineering, vol. 2, no. 3 (2014): 62-66. doi: 10.12691/ajeee-2-3-1.

\section{Introduction}

Microstrip antenna is a simple patch antenna which consists of radiated patch component, dielectric substrate, and ground plane. The radiated patch and ground plane is a thin layer of copper or gold which is a perfect electric conductor. dielectric substrate has its own dielectric permittivity values which influences the size of the Antenna. Bottom layer of dielectric substrate is fully covered by conductors that act as a ground plane. The thickness of substrates layer generate surface wave with low propagation that cause loss of power, but can increase the bandwidth and efficiency. Microstrip antenna has several advantages like low profile antenna, light weight, small dimension, cheap and easy to integrate with other circuits which make it suitable for many applications [1].

Fractal Antennas Elements are currently beneficial to antenna designers \& researchers. By applying fractals to antenna elements:

1. Antennas with smaller size can be created.

2. Resonance frequencies that are multiband can be achieved.

3. Optimization for directivity \& gain.

4. Wideband frequency band can be achieved.

Most fractals have infinite complexity and detail that can be used to reduce antenna size and develop low profile antennas. For most fractals, self-similarity concept can achieve multiple frequency bands because of different parts of the antenna are similar to each other at different scales. The combination of infinite complexity and self similarity makes it possible to design antennas with various wideband performances [2].

\section{Fractal Theory}

In modern wireless communication systems wider bandwidth, multiband and low profile antennas are in great demand for various communication applications. This has initiated antenna research in various directions, one of them is using fractal shaped antenna elements. Fractal shaped antennas has various unique characteristics that are linked to the various geometry and properties of fractals. Fractals were first defined by Benoit Mandelbrot in 1975 as a way of classifying structures whose dimensions were not whole numbers. Fractal geometry has unique geometrical features occurring in nature. It can be used to describe the branching of tree leaves and plants, rough terrain, jaggedness of coastline, and many more examples in nature. Fractals have been applied in various field like image compression, analysis of high altitude lightning phenomena, and rapid studies are apply to creating new type of antennas. Fractals are geometric forms that can be found in nature, being obtained after millions of years of evolution, selection and optimization [3]. 
The increase of wireless technology has motivated the designers to make new antenna design that can cover wide range of frequencies and can be useful for multipurpose. Due to the low cost, low profile and complex configuration it has gained interest for its application. Here we have designed an antenna that will be useful for multiband application. Fractal geometry is used for designing antenna that has been successfully applied to get wide range of bandwidth. Here, for designing of this antenna we use Sierpinski Carpet fractal geometry. A dual band antenna using a Sierpinski carpet fractal geometry is designed which covers the multiband characteristics of fractals [4].

Fractal antenna is widely used due to the following important facts [5]:

1 . Very broadband and multiband frequency response that derives from the inherent properties of the fractal geometry of the antenna.

2. Compact size compared to antennas of conventional designs, while maintaining good to excellent efficiencies and gains.

3. Mechanical simplicity and robustness; the characteristics of the fractal antenna are obtained due to its geometry and not by the addition of discrete components.

4. Design to particular multi frequency characteristics containing specified stop bands as well as specific multiple pass bands.

\section{Fractal Geometry}

There are many fractal geometries that have been found to be useful in developing new and innovative design for antennas [6]. Figure below shows some of these unique geometries.

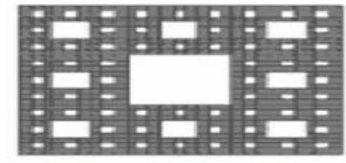

Sierpinski carpet

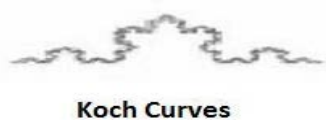

Figure 3.1. Some unique fractal geometry

\section{Antenna Design Specification}

Here the proposed Sierpinski Carpet antenna is designed from a simple squre patch of dimension of $\mathrm{L} x$ $\mathrm{W} \times \mathrm{h}=36 \mathrm{~mm} \times 36 \mathrm{~mm} \times 1 \mathrm{~mm}$. The substrate is with a dielectric constant of $\varepsilon_{\mathrm{r}}=4.2$. Multiple iterations are designed by using IFS. In order to start the proposed fractal antenna, it begins with a square patch, and then divides it into nine smaller congruent squares where the open central square is dropped. The remaining eight squares are divided into nine smaller congruent square. The construction of the proposed fractal shape is carried out by applying a finite number of times an iterative process performed on a simple starting topology [7]. According to the properties of self similarity, the fractal dimension Ds of a set $\mathrm{A}$ is defined as:

$$
\text { Ds }=\log (\mathrm{N}) / \log (\mathrm{r})
$$

Where $\mathrm{N}$ is the total number of distinct copies similar to $\mathrm{A}$, and $\mathrm{A}$ is scaled down by a ratio of $1 / \mathrm{r}$.

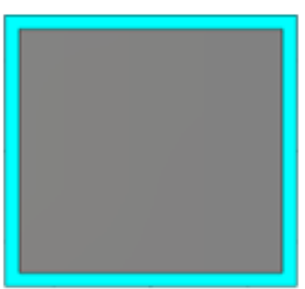

Figure la. Stage 0

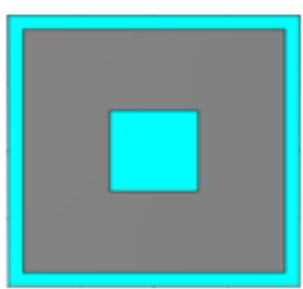

Figure 1b. Stage 1

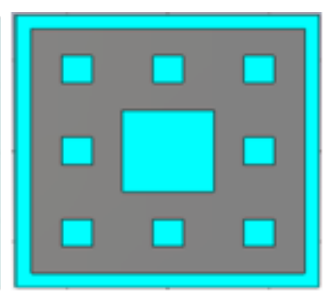

Figure lc. Stage 2

Figure 4.1. Steps of Iteration to get Carpet geometry

\section{Simulation Results \& Analysis}

\section{$0^{\text {th }}$ ITERATION}

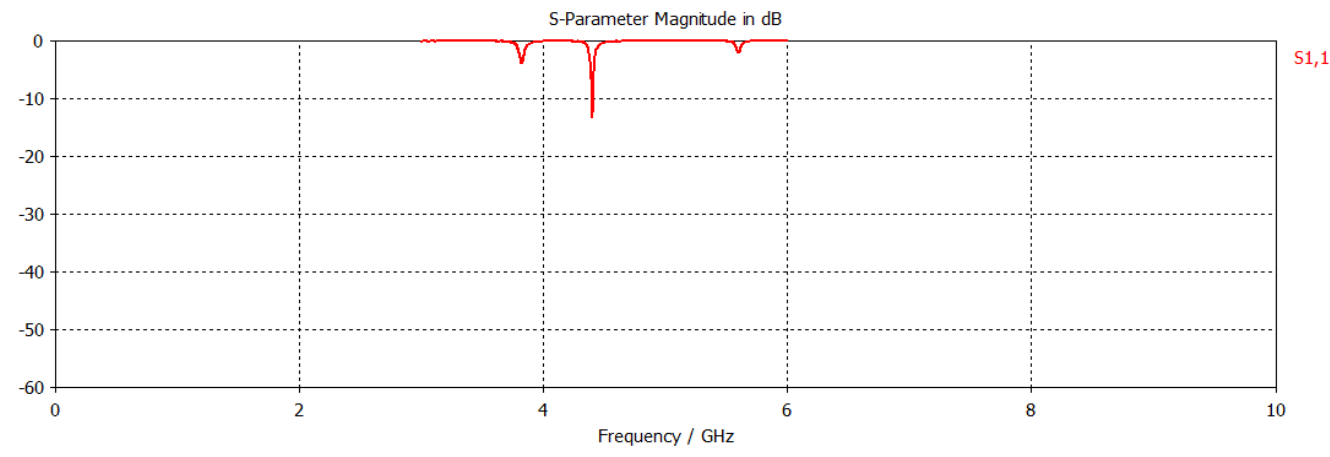

Figure 5.1. Simulated reflection coefficient (S11) with frequency 


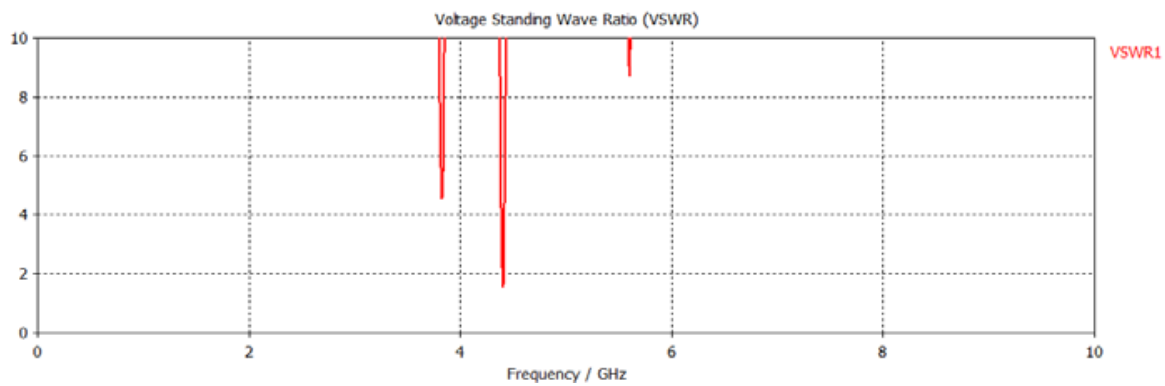

Figure 5.2. Variation of VSWR with frequency

\section{$1^{\text {st }}$ ITERATION}

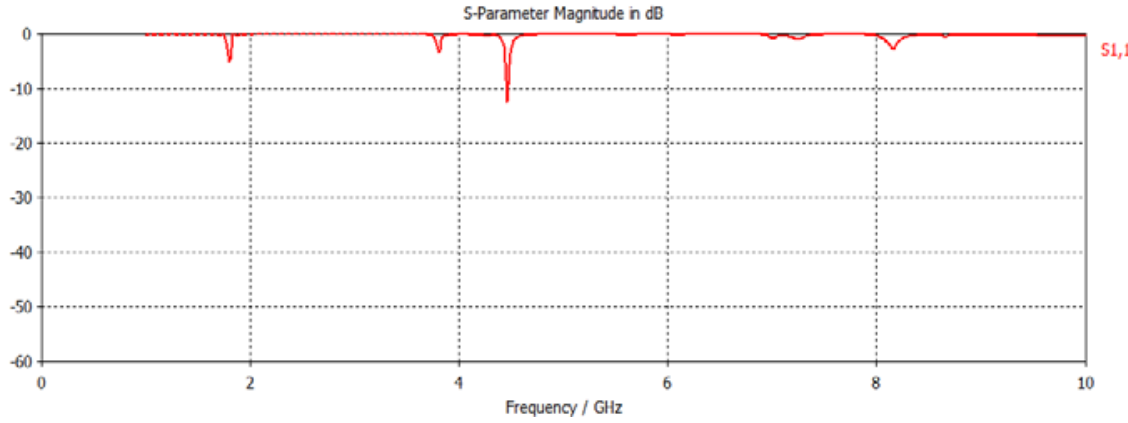

Figure 5.3. Simulated reflection coefficient (S11) with frequency

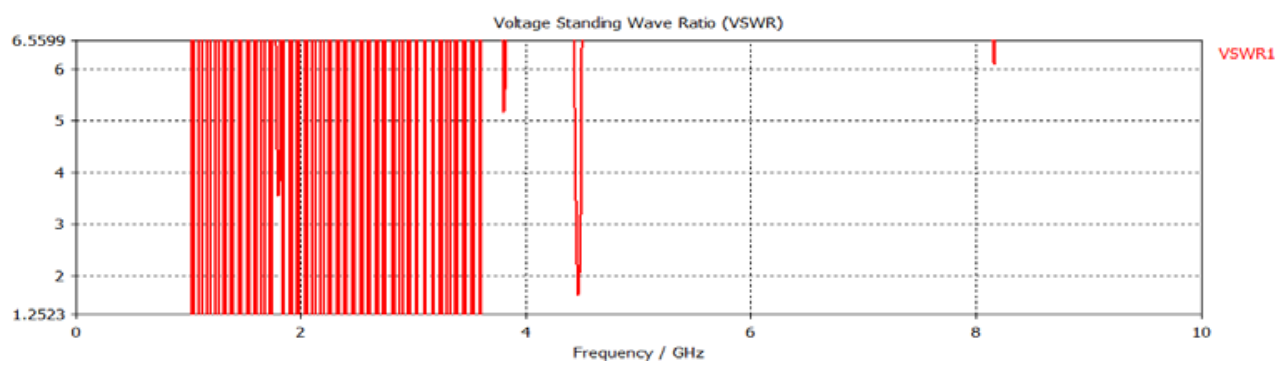

Figure 5.4. Variation of VSWR with frequency

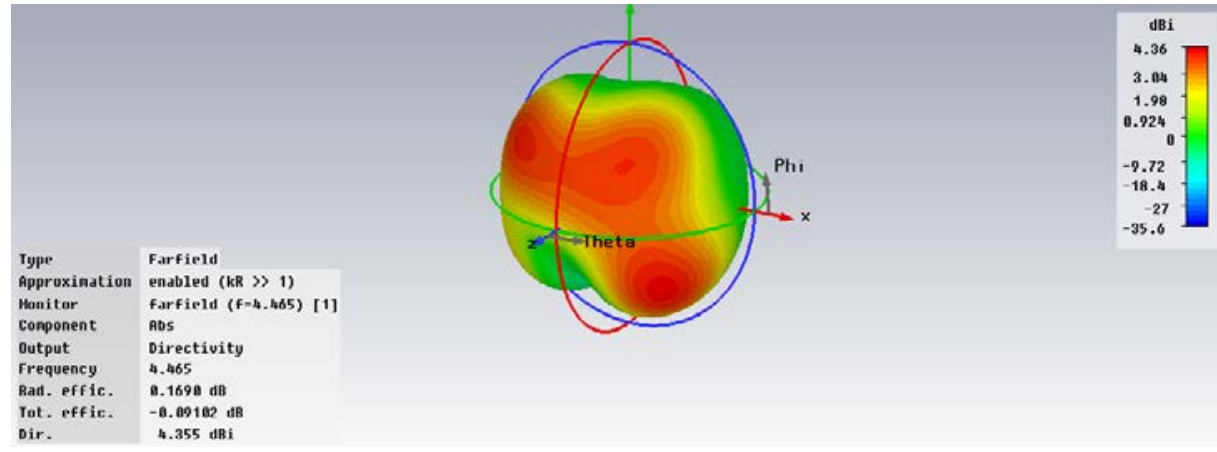

Figure 5.5. 3D plot for directivity at $8.551 \mathrm{GHz}$

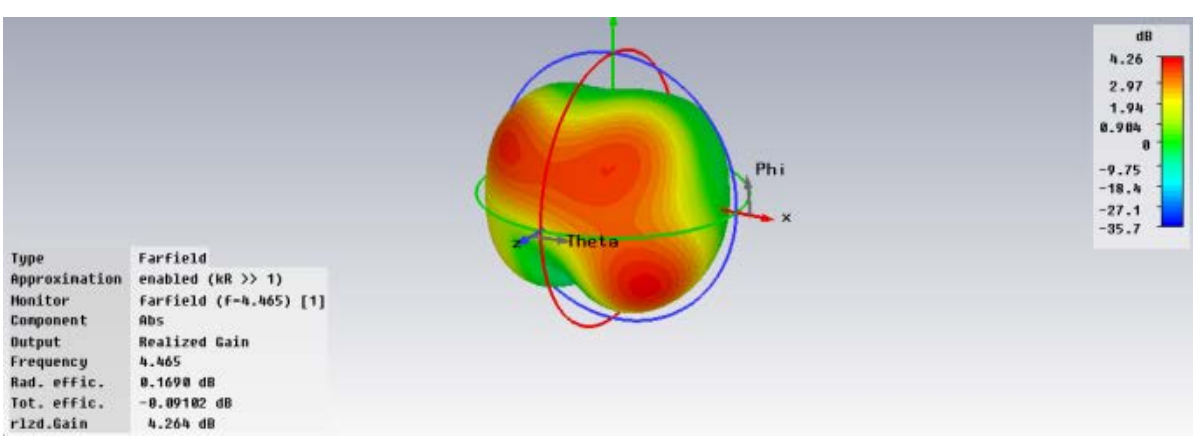

Figure 5.6. 3D plot for realized gain at $8.551 \mathrm{GHz}$ 


\section{$2^{\text {nd }}$ ITERATION}

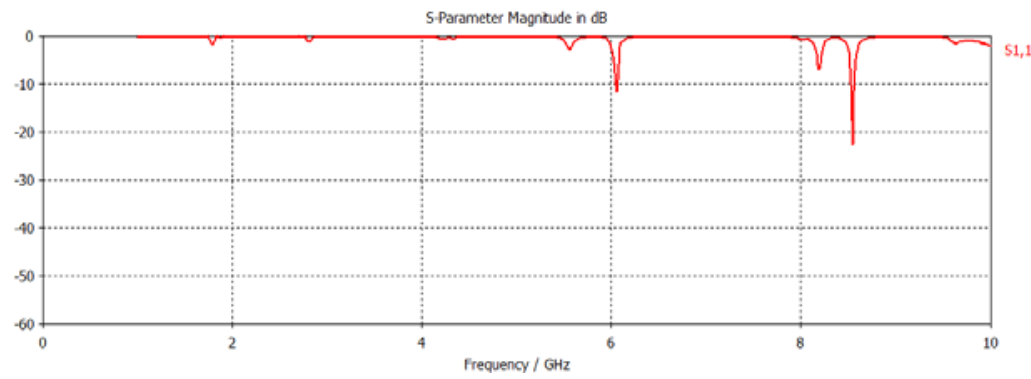

Figure 5.7. Simulated reflection coefficient (S11) with frequency

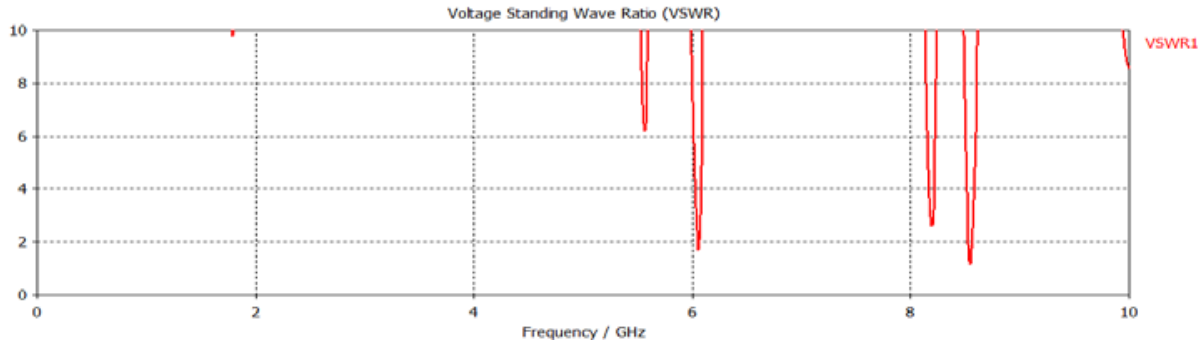

Figure 5.8. Variation of VSWR with frequency

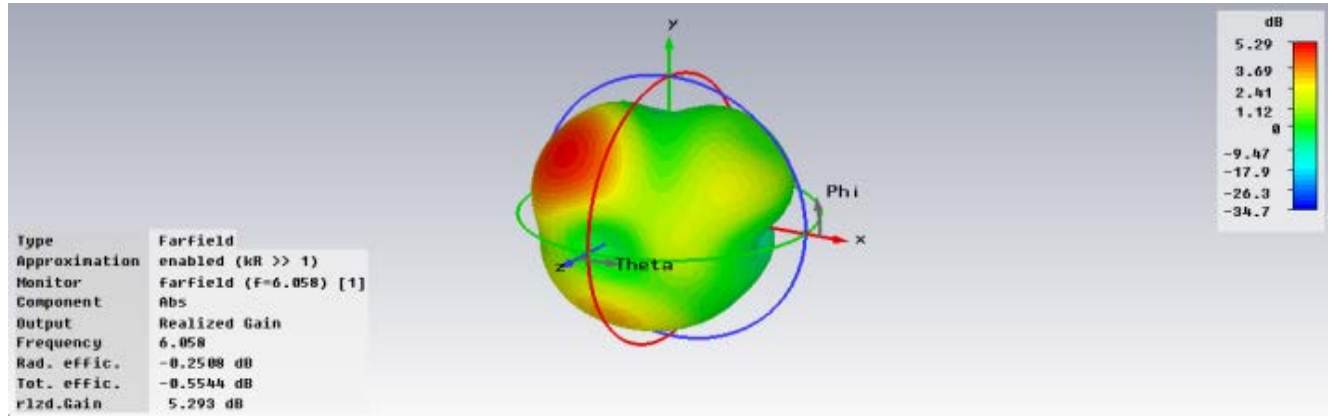

Figure 5.9. 3D plot for realized gain at $6.058 \mathrm{GHz}$

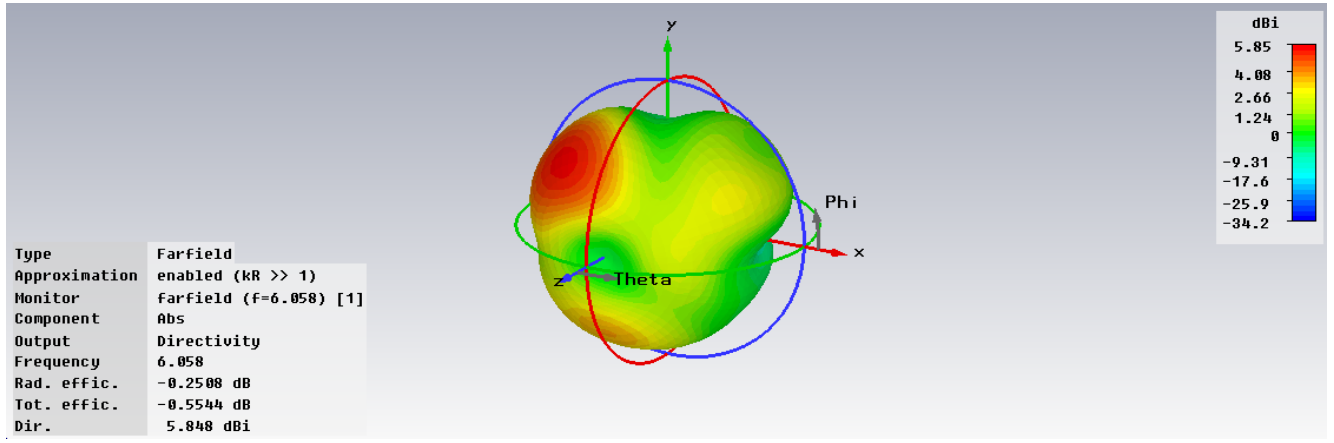

Figure 5.10. 3D plot for directivity at $6.058 \mathrm{GHz}$

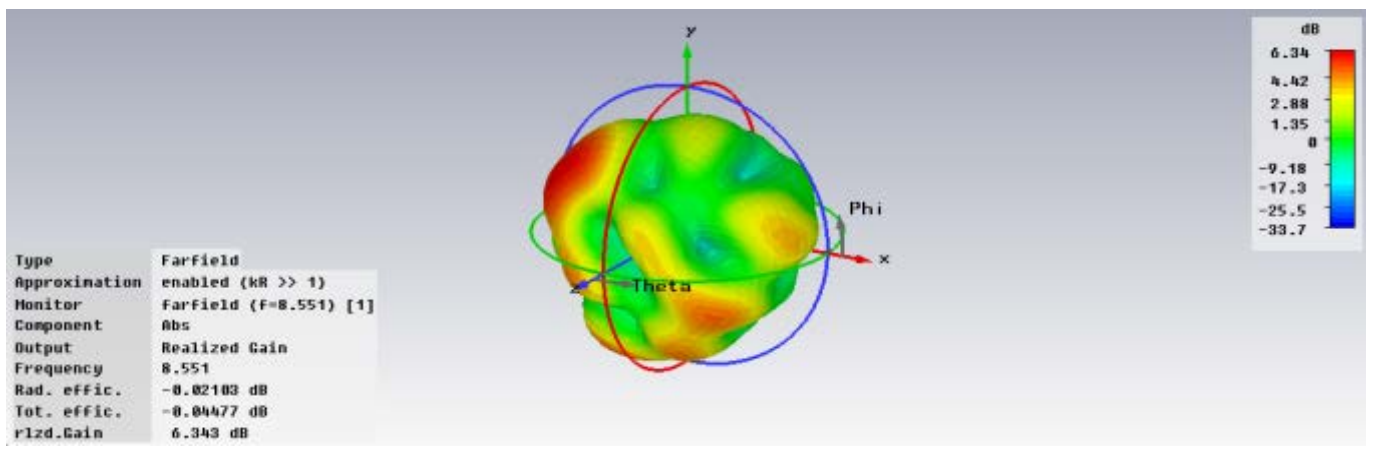

Figure 5.11. 3D plot for realized gain at $8.551 \mathrm{GHz}$ 


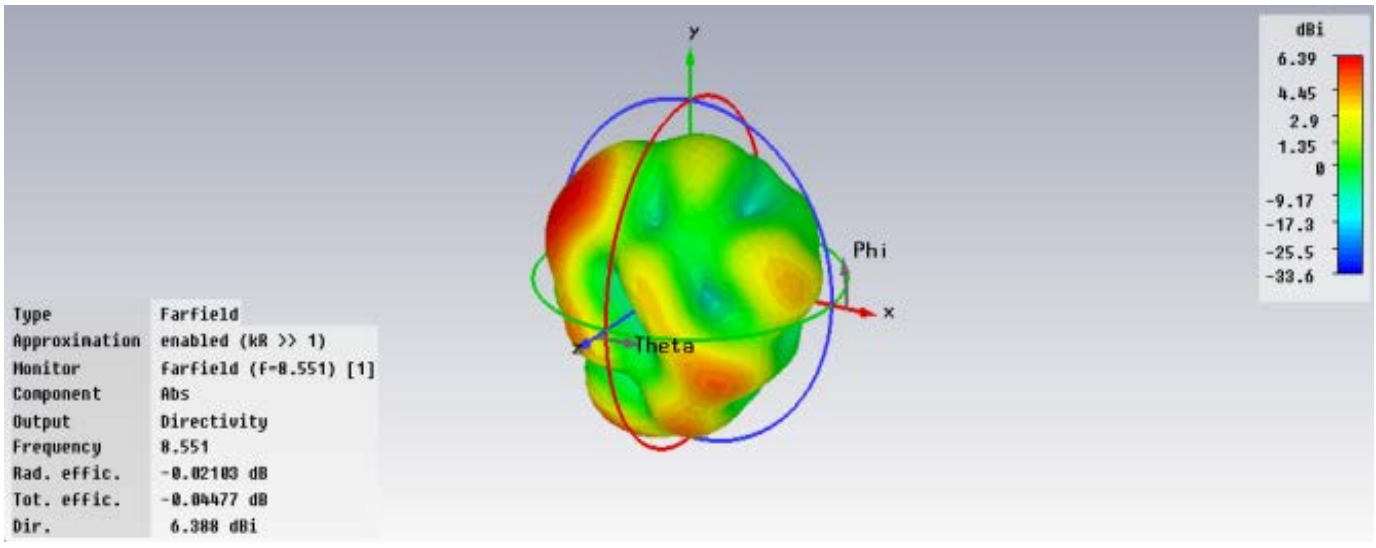

Figure 5.12. 3D plot for directivity at $8.551 \mathrm{GHz}$

\section{Calculation for Enhanced Bandwidth of Increasing Iteration}

\section{$0^{\text {th }}$ ITERATION:}

$\mathrm{f}_{\mathrm{l}}=4.3998 \mathrm{Ghz}, \mathrm{f}_{\mathrm{h}}=4.4095 \mathrm{Ghz} \frac{\left(f_{h}-f_{l}\right)}{\mathrm{f}_{\mathrm{r}}} \times 100=$ $0.22 \%$

$1^{\text {st }}$ ITERATION:

$\mathrm{f}_{\mathrm{l}}=4.4596 \mathrm{Ghz}, \mathrm{f}_{\mathrm{h}}=4.472 \mathrm{Ghz} \& \mathrm{f}_{\mathrm{r}}=4.465 \mathrm{Ghz}$
Bandwidth enhancement $=\frac{\left(f_{h}-f_{l}\right)}{\mathrm{f}_{\mathrm{r}}} \times 100=0.22 \%$

$2^{\text {nd }}$ ITERATION:

For band 1

$\mathrm{f}_{\mathrm{l}}=6.0485 \mathrm{Ghz}$, fh $=6.0642 \mathrm{Ghz} \& \mathrm{fr}=6.058 \mathrm{Ghz}$

Bandwidth enhancement $=\frac{\left(f_{h}-f_{l}\right)}{\mathrm{f}_{\mathrm{r}}} \times 100=0.25 \%$

For band 2

$\mathrm{f}_{\mathrm{l}}=8.5238 \mathrm{Ghz}$, fh $=8.5632 \mathrm{Ghz} \& \mathrm{fr}=8.551 \mathrm{Ghz}$

Bandwidth enhancement $=\frac{\left(f_{h}-f_{l}\right)}{f_{\mathrm{r}}} \times 100=0.46 \%$

Table 1. Comparison of various performance parameters for multiple iterations

\begin{tabular}{|c|c|c|c|c|c|c|}
\hline Iterations & Resonant frequency $(\mathrm{GHz})$ & Return losses (dB) & VSWR & Directivity (dBi) & Gain (dB) & Bandwidth (GHz) \\
\hline 0 & 4.404 & -11.129 & 1.76 & 4.10 & 4.10 & .20 \\
\hline $1^{\text {st }}$ & 4.465 & -12.358 & 1.635 & 4.35 & 4.35 & .22 \\
\hline \multirow{2}{*}{$2^{\text {nd }}$} & 6.058 & -11.7 & 1.55 & 5.29 & 5.29 & .25 \\
\hline & 8.551 & -22.637 & 1.15 & 6.34 & 6.34 & .46 \\
\hline
\end{tabular}

\section{Conclusion}

The proposed Sierpinski Carpet fractal antenna upto 2nd iteration is built \& simulated using the CST Microwave Studio Suite. It is observed that with increase in the number of iterations the bandwidth of the antenna increases \& on second iterations the antenna starts showing the multiband behavior. Increase in multiple iterations also led to improvement in various performance parameters like VSWR, directivity, gain \& return losses. The simulated results shows good band width enhancement. The Sierpinski Carpet fractal antenna is observed to possess multiband behavior, hence can be suggested for satellite communication.

\section{Acknowledgement}

The authors sincerely thank to the Vice Chancellor, the H.O.D of EL\&TCE Dept. VSSUT, BURLA for constant encouragement and support. Authors are thankful to all the staff of EL\&TCE Dept. VSSUT,BURLA for their support directly or indirectly.

\section{References}

[1] Constantine A. Balanis, Antenna Theory Analysis \& Design, John Wiley \& Sons, 1997.

[2] G Vinoy, K. J., "Fractal shaped antenna elements for wide and multi-band wireless applications,” Thesis, Pennsylvania, Aug. 2002.

[3] B. B. Mandlebrot, "The Fractal Geometry of Nature”, New York: W.H. Freeman, 1983.

[4] Ghatak, R.; Mishra, R.K.; Poddar, D.R., "Perturbed Sierpinski Carpet Antenna with CPW Feed for IEEE 802. 11 a/b WLAN Application," Antennas and Wireless Propagation Letters, IEEE, vol.7, no., pp. 742-744, 2008.

[5] B.-L. Ooi, "A modified contour integral analysis for Sierpinski fractal carpet antennas with and without electromagnetic band gap groundplane,” IEEE Trans. Antennas Propag., vol. 52, pp. 12861293, May 2004.

[6] J. Gutrman, A. A. Moreira, and C. Peixeiro, "Microstrip fractal antennas for multistandard terminals,” IEEE Antennas Wireless Propag.Lett., vol. 3, pp. 351-2004.

[7] C. Puente, J. Romeu, R. Pous, and A. Cardama, "On the behavior of the Sierpinski multiband fractal antenna," IEEE Trans. Antennas Propagat., vol. 46, pp. 517-524, Apr. 1998. 